\title{
The lateral effect research of traffic flow based on the modified car- following model
}

\author{
Jie-Yin ZHENG ${ }^{1, a}$, Ya-Zhou ZHENG ${ }^{1, b}$ and Hong-Xia GE ${ }^{1,2,3, c}$ \\ 1. Ningbo University, Ningbo, 315211, China \\ 2. Jiangsu Key Laboratory of Urban ITS, Southeast University, Nanjing 210096, China \\ 3. National Traffic Management Engineering and Technology Research Centre Ningbo University \\ Sub-centre, Ningbo 315211, China \\ a) guangyuxinkong@126.com; b) zhengyazhoumath@163.com; c) gehongxia@nbu.edu.cn \\ *Corresponding author :gehongxia@nbu.edu.cn
}

Keywords: car-following model; stability condition; feedback control method

\begin{abstract}
In this paper, an improved car-following model is proposed to suppress the traffic jams. Considering the relative velocity, relative optimal velocity and the difference between safety distance and headway, a comprehensive control scheme is constructed according to the feedback control theory. The stability condition for the modified model is obtained by using the linear stability theory. Numerical simulation is carried out to illustrate the advantage of our model with the new control signal, and the results are in good accordance with the theoretical analysis.
\end{abstract}

\section{Introduction}

In recent decades, traffic problems have influenced human's daily life, such as the traffic accident, fuel consumption and pollution. In order to solve those increasingly serious traffic problems, many traffic models have been put forward to study the complex phenomena of traffic jams. What's more, the scientific researchers have obtained many significant results [1-3].

To date, the problem of traffic jam has attracted much attention in the study of traffic flow. The concept of car-following was first described by Reuschel and Pipes [4], which assumed that the vehicle behind adjusts its behavior with respect to the preceding vehicle in the same lane. In1961, Newell [5] developed a car-following model with a differential equation and gave a graphic description of the optimal velocity (OV) function. In 1995, Bando et al. [6] proposed a microscopic traffic model called optimal velocity model (OVM). Further, Konishi et al. [9] proposed a chaotic car-following model by setting the time delay feedback control schemes, and analyzed theoretically the traffic jam phenomena for open flow in 1999. Later, some scholars extended the model by introducing multiple information into relative velocity or headway [7].

So far, there is just little research of car-following from the viewpoint of control methods. In real traffic flow, on urban road without isolation belts, it is necessary to consider the effects of vehicles on adjacent lanes, and we need to taken into account the headway between the proceeding and following vehicles.

The rest of this paper is organized as follows. The car-following model considering the lateral effect is presented in section 2. In Section 3, the modified model including a new feedback control signal is constructed and the feedback control method is used to analyze the stability conditions. In Section 4, numerical simulation is carried out to confirm the theoretical results. Conclusions are given in Section 5.

\section{Car-following model}

To investigate the effects of the non-motor vehicles on adjacent lanes without isolation belts, the car-following model is depicted as follows: 
$\frac{d^{2} x_{n}(t)}{d t^{2}}=a\left[V^{o p}\left(\Delta x_{n}(t)\right)+\kappa\left(p \bar{V}^{o p}\left(w_{n}(t)\right)+q \bar{V}^{o p}\left(l_{n}(t)\right)\right)\right] \quad-a v_{n}(t)$

(1)

where $\Delta X_{n}(t)=X_{n+1}(t)-x_{n}(t), \Delta v_{n}(t)=v_{n+1}(t)-v_{n}(t)$ are the headway and the velocity difference between the $n$ - $t$ vehicle and the preceding vehicle, respectively; $x_{n}(t)$ is the $n$-th vehicle's position, $a$ is the sensitivity of driver and $k$ is the influence coefficient between motor vehicle and non-motor vehicle; $V^{o p}\left(\Delta x_{n}(t)\right)$ is the optimal velocity function. The OV function is expressed as follows:

$V^{o p}\left(\Delta x_{n}(t)\right)=\frac{v_{\max }}{2}\left[\tanh \left(\Delta x_{n}(t)-h_{c}\right)+\tanh \left(h_{c}\right)\right](2) \bar{V}^{o p}\left(w_{n}(t)\right)=\left\{\begin{array}{l}0, \quad \bar{x}_{n} \geq d_{1}, \\ V^{o p}\left(w_{n}(t)\right), \quad 0 \leq \bar{x}_{n}<d_{1},\end{array}\right.$

$\bar{V}^{o p}\left(l_{n}(t)\right)=\left\{\begin{array}{l}0, \quad \bar{X}_{n}>d_{2}, \\ V^{o p}\left(l_{n}(t)\right), \quad 0 \leq \bar{X}_{n}<d_{2},\end{array}\right.$

where ${ }^{\bar{X}}$ is the distance between the $n$-th motor vehicle and the non-motor vehicle, and $\bar{X}_{n}$ is the minimum lateral distance between the $n$-th motor and non-motor vehicles; ${ }{ }_{\max }$ is the maximum velocity and $h_{c}$ is the safety headway distance; $w_{n}=\bar{x}_{n}-d_{1}, l_{n}=\bar{x}_{n}-d_{2} \cdot d_{1}, d_{2}$ are the lateral and longitudinal safety distances respectively; $p, q(p+q=1)$ are the reaction coefficients of the lateral and longitudinal distances respectively.

In fact, according to the control theories, we have studied the stability condition for the system (1), which is

$0 \leq \mathrm{K} \leq \sqrt{\frac{a-2 \Lambda_{1}}{a\left[\left(p \Lambda_{2}\right)^{2}+\left(q \Lambda_{3}\right)^{2}\right]}},\left(a-2 \Lambda_{1} \geq 0\right)$.

where $\Lambda_{1}=\frac{d V^{o p}\left(y_{n}(t)\right)}{d y_{n}(t)} \mid y_{n}(t)=V^{o p-1}\left(v_{0}\right)$;

$$
\begin{gathered}
\Lambda_{2}=\frac{d \bar{V}^{o p}\left(w_{n}(t)\right)}{d w_{n}(t)} \mid w_{n}(t)=d_{1}^{*} ; \\
\Lambda_{3}=\frac{d \bar{V}^{o p}\left(l_{n}(t)\right)}{d_{n}(t)} \mid l_{n}(t)=d_{2}^{*} \text { and } y_{n}(t)=\Delta x_{n}(t) .
\end{gathered}
$$

\section{Control scheme}

Considering the positive and negative velocity differences and optimal velocity differences into account, in the meantime, in order to avoid collision, the difference between the safety headway and the headway is considered. The new feedback control signal $u_{n}(t)$ including all these factors is added into system (1), so we have

$$
\frac{d^{2} x_{n}(t)}{d t^{2}}=a\left[V^{o p}\left(\Delta x_{n}(t)\right)+\kappa\left(p \bar{V}^{o p}\left(w_{n}(t)\right)+q \bar{V}^{o p}\left(l_{n}(t)\right)\right)\right] \quad-a v_{n}(t)+u_{n}(t)
$$

$u_{n}(t)=\gamma\left[\Delta v_{n}(t)+\Delta v_{n}^{o p}(t)\right]-\lambda^{2}\left(h_{c}-y_{n}(t)\right)$. 
where $\gamma(\gamma>0)$ is the reaction coefficient for the relative velocity $\Delta V_{n}(t)$ and the relative optimal velocity $\Delta V_{n}^{o p}(t) ; \lambda$ is another reaction coefficient for the $H\left(y_{n}(t)-h_{c}\right)\left(h_{c}-y_{n}(t)\right)$.

Then, Eq. (6) can be described as

$$
\left\{\begin{array}{l}
\frac{d v_{n}(t)}{d t}=a\left[V^{o p}\left(\Delta y_{n}(t)\right)+\kappa\left(p \bar{V}^{o p}\left(w_{n}(t)\right)+q \bar{V}^{o p}\left(l_{n}(t)\right)\right)\right] \\
-a v_{n}(t)+\gamma\left[v_{n+1}(t)-v_{n}(t)+\left(V^{o p}\left(y_{n+1}(t)-V^{o p}\left(y_{n}(t)\right)\right)\right)\right](8) \\
-\lambda^{2}\left(h_{c}-y_{n}(t)\right) \\
\frac{d v_{n}(t)}{d t}=v_{n+1}(t)-v_{n}(t) .
\end{array}\right.
$$

at a constant velocity $v_{0}$, so we can get the the steady state for the following vehicles, that is $\left[v_{n}^{*}(t), y_{n}^{*}(t)\right]^{T}=\left[v_{0}, V^{o p-1}\left(v_{0}\right)\right]^{T}$.

The traffic system (8) can be linearized at the steady state

(9), that is

$$
\begin{aligned}
& \left\{\begin{array}{l}
\frac{d \delta v_{n}(t)}{d t}=a\left[\delta y_{n}(t) \Lambda_{1}+\kappa\left(p \delta w_{n}(t) \Lambda_{2}+q \delta l_{n}(t) \Lambda_{3}\right)\right] \\
-\delta v_{n}(t)+\gamma\left[\delta v_{n+1}(t)-\delta v_{n}(t)+\left(\delta\left(y_{n+1}(t) \Lambda_{4}-\delta y_{n}(t) \Lambda_{1}\right)\right)\right] \\
+\lambda^{2} \delta y_{n}(t) \\
\frac{d \delta v_{n}(t)}{d t}=\delta v_{n+1}(t)-\delta v_{n}(t) .
\end{array}\right. \\
& \delta v_{n}(t)=v_{n}(t)-v_{0}, \delta y_{n}(t)=y_{n}(t)-V^{o p-1}\left(v_{0}\right) \\
& \quad \delta w_{n}(t)=\delta \bar{x}_{n}-d_{1}^{*}, \delta l_{n}=\delta \bar{x}_{n}-d_{2}^{*} \\
& \quad \Lambda_{4}=\frac{d V^{o p}\left(y_{n+1}(t)\right)}{d y_{n+1}(t)} \mid y_{n+1}(t)=V^{o p-1}\left(v_{0}\right) .
\end{aligned}
$$

where

After the Laplace transform for Eq.(12), we can get the transfer function $\bar{G}(s)$ as follows:

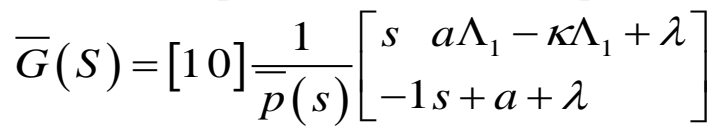

$$
\begin{aligned}
& \times\left[\begin{array}{lccl}
a \kappa p \Lambda_{2} & a \kappa p \Lambda_{3} \gamma & \gamma \Lambda_{4} \\
0 & 0 & 1 & 0
\end{array}\right] \\
& =\frac{1}{\bar{p}(s)}\left[s \kappa p \Lambda_{2} \quad s \kappa q \Lambda_{2} \quad s \gamma+\beta \quad s \gamma \Lambda_{4}\right](11) \\
& \bar{p}(s)=s^{2}+\alpha s+\beta \text {, } \\
& \alpha=a+\gamma, \beta=(a-\kappa) \Lambda_{1}+\lambda^{2}
\end{aligned}
$$

where $S$ is a complex variable.

Based on the control theories, traffic jams will never

occur in the traffic flow system as long as the characteristic function $\bar{p}(s)$ is stable and $\|\bar{G}(s)\|_{\infty} \leq 1$. According to the Hurwitz stability criterion, it is easy to obtain that the characteristic function $\bar{p}(s)$ is stable. Then, consider $\|\bar{G}(s)\|_{\infty} \leq 1$, that is

$\|\bar{G}(s)\|_{\infty}=\sup _{\omega \in[0, \infty)}|\bar{G}(j \omega)| \leq 1$ 


$$
\begin{aligned}
& |\bar{G}(j \omega)|^{2}=|\bar{G}(-j \omega) \bar{G}(j \omega)| \\
& =\frac{(a \kappa p \omega)^{2}+(a \kappa q \omega)^{2}+(\lambda \omega)^{2}+\beta^{2}+\left(\gamma \Lambda_{4} \omega\right)^{2}}{\left(\beta-\omega^{2}\right)+(\alpha \omega)^{2}} \leq 1 .
\end{aligned}
$$

Then, we can obtain the sufficient condition through the analysis above. We have the region for the reaction $\kappa$

$0 \leq \kappa \leq \sqrt{\frac{a^{2}+2 a \gamma-2 a \Lambda_{1}-2 \lambda^{2}-\left(\lambda \Lambda_{4}\right)^{2}}{a\left[\left(p \Lambda_{2}\right)^{2}+\left(q \Lambda_{3}\right)^{2}\right]}}$,

where $a^{2}+2 a \gamma-2 a \Lambda_{1}-2 \lambda^{2}-\left(\lambda \Lambda_{4}\right)^{2} \geq 0$.

\section{Numerical simulation}

In the section, the parameters for the modified car-following model are set as $h_{c}=7.02 \mathrm{~m}, a=$ $2 \mathrm{~s}^{-1}, d_{1}=5.5 \mathrm{~m}, d_{2}=6.5 \mathrm{~m}, v_{0}=20 \mathrm{~m} / \mathrm{s}$ and $T=0.1 \mathrm{~s}$. It is assumed that all vehicles have the same parameters. The initial condition is the steady state for the model, and the initial positions and speeds are set as $x_{n}(0)=\sum y_{n}^{*}, y_{n}(0)=y_{n}^{*}(t), v_{n}(0)=v_{n}^{*}(t) n=1,2,3, \ldots, N$, and $N=120$ is the total number of vehicles. We consider a case where the leading vehicle stops suddenly for $v_{n}(0)=0$, $t=n T=100-103$.

Figure 1 shows the velocity-time patterns of the 1th, the 25th and the 50th vehicles with different parameter values of $\gamma$. It can be seen from Fig. 1 that with the control signal, as the reaction coefficient $\gamma$ increases from 0.15 to 0.75 , the stability of the traffic system is strengthened. And we can find that vehicles can reach steady running state in relatively short time with the increasing of reaction coefficient $\gamma$.
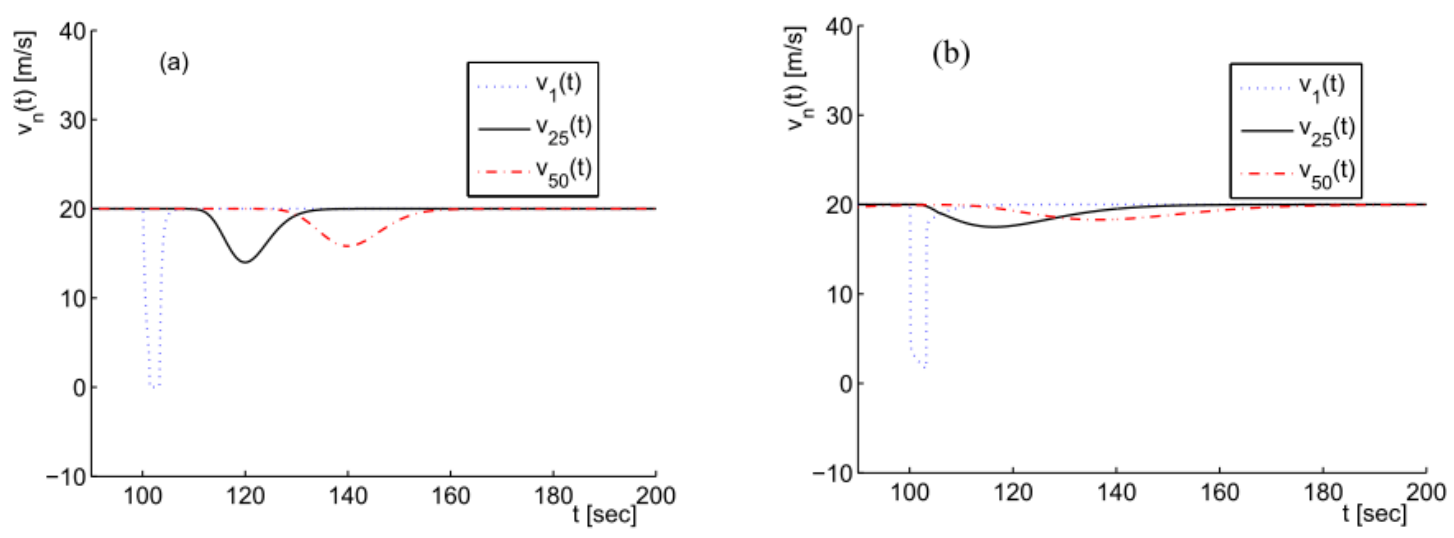

Figure 1. Numerical simulations for the modified car-following model with (a) $\gamma=0.15$,(b) $\gamma=0.75$.

Now consider the system with the new control signal. As the stability condition in Eq. (5) and Eq. (15) is met, the comparisons between the results in Fig. 2 and Fig. 3 are carried out. When we choose the appropriate parameters $\left(\kappa=0.75, \gamma=0.75, \lambda=0.48, \nu_{\max }=33.6 \mathrm{~m} / \mathrm{s}\right)$, we can find that vehicles can reach more steady running state in relatively short time, although the maximal speed is larger compared with Fig. 2. The amplitude of the velocity for the 25th vehicle decreases and the 50th vehicle runs more smoothly. That is to say, as we add a feedback control signal to system (1), the traffic flow can reach steady state quickly. 


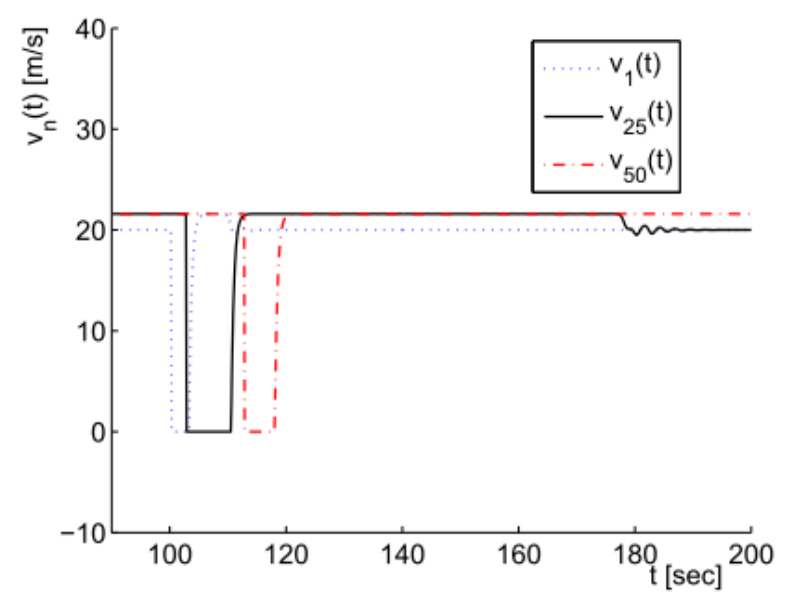

(2a)

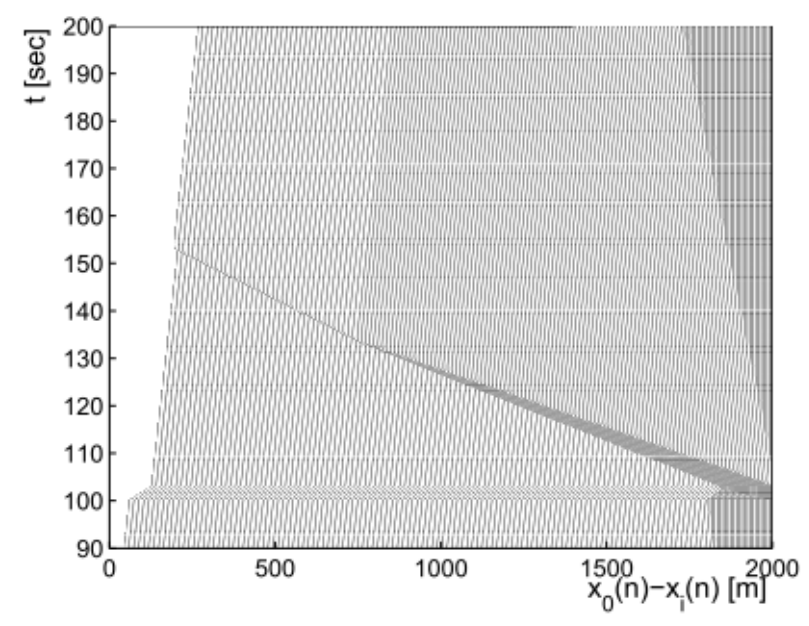

(2b)

Figure 2. (2a) Space-time plot of the traffic system . (2b) Temporal velocity behavior of the first, 25th and 50th vehicles. $\left(\kappa=0.5, \lambda=0, \gamma=0, v_{\max }=21.5 \mathrm{~m} / \mathrm{s}\right)$

From the above simulation, it can be concluded that the proposed feedback control scheme is useful for suppressing the traffic congestion problems.

\section{Conclusions}

In this study, a improved car-following model considering the lateral effect has been proposed. The other effects of some important comprehensive information on the traffic current and the jamming transition have been investigated. Moreover, the stability condition has been obtained for the model by using control method. The new model has been tested by using numerical simulations and the results are consistent with theoretical ones. 


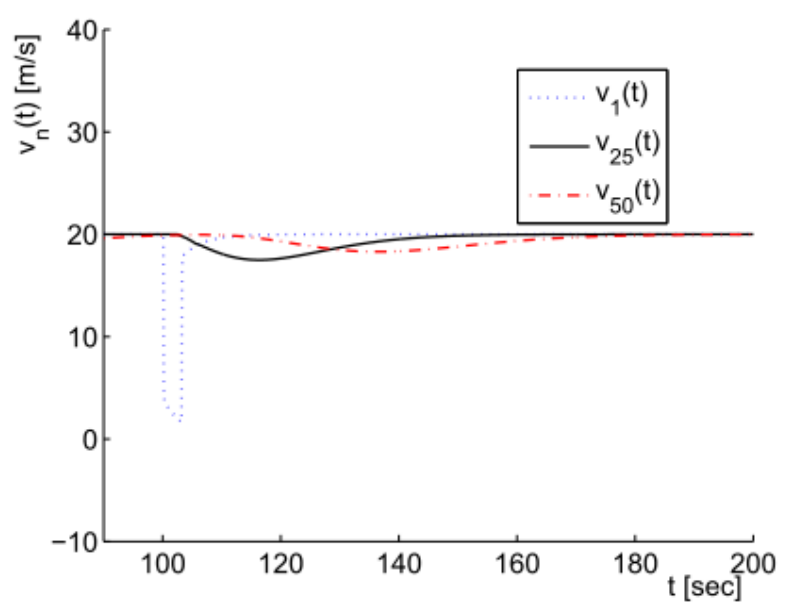

(3a)

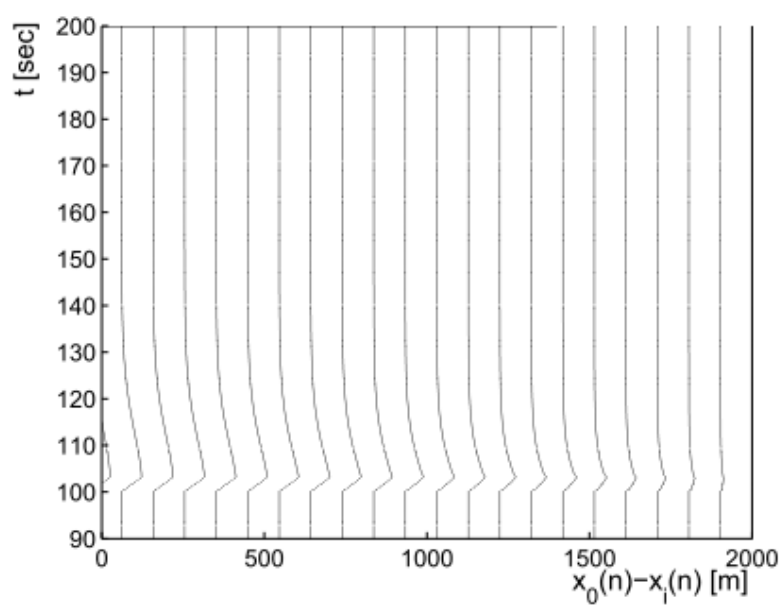

(3b)

Figure 3. (3a) Space-time plot of the traffic system . (3b) Temporal velocity behavior of the first, 25th and 50th vehicles. ( $\kappa=0.75, \lambda=0.48, \gamma=0.75, v_{\max }=33.6 \mathrm{~m} / \mathrm{s}$ )

\section{Acknowledgments}

The project supported by the Science Foundation of Ningbo (Grant Nos. 2014A610022 and 2014A6111015), Humanities and Social Science Project of Ministry of Education of China (Grant No.14jyc630171), Soft Science Project of Zhejiang Province (Grant No.2014C35076) the Open Research Fund of Jiangsu Key laboratory of Urban ITS, Southeast University and the K. C. Wong Magna Fund in Ningbo University, China.

\section{References}

[1] B. S. Kerner, and H. Rehborn, "Experimental properties of complexity in traffic flow," Phys. Rev. E, 53: 1297, 1996.

[2] T. Q. Tang, J. G. Li, Y. P. Wang and G. Z. Yu, "Vehicles fuel consumption of car-following models,” Science China Technological Sciences, 56(5): 1307, 2013.

[3] T. Nagatani. Modified KdV equation for jamming transition in the continuum models of traffic. Phys. A, 261: 599, 1998.

[4] L. A. Pipes, “An Operational Analysis of Traffic Dynamics,” Journal of Applied Physics, 24(3): 274, 1953. 
[5] G. F. Newell, "Nonlinear Effects in the Dynamics of Car Following," Operations research, 9: 209, 1961.

[6] M. Bando, K. Hasebe, A.Nakayama, A. Shibata and Y. Sugiyama, "Dynamical model of traffic congestion and numerical simulation,” Phys. Rev. E, 58: 5429, 1995.

[7] H. X. Ge, S. Q. Dai, Y. Xue and L. Y. Dong, "Stabilization analysis and modified Korteweg-de Vries equation in a cooperative driving system,” Phys Rev. E, 71: 066119, 2005.

[8] G. H. Peng and D. H. Sun, “A dynamical model of car-following with the consideration of the multiple information of preceding cars, Physics Letters A, 374: 1694, 2010.

[9] K. Konishi, H. Kokame and K. Hirata, "Decentralized delayed-feedback control of an optimal velocity traffic model,” The European Physical Journal B, 15: 715, 2000. 